\title{
Alterações quantitativas do filme lacrimal após cirurgia refrativa: estudo comparativo entre PRK e LASIK
}

\author{
Quantitativechanges in tear film after refractive surgery:comparative study \\ between PRK and LASIK
}

\author{
Marcelo Galiano Credie ${ }^{1}$ \\ Maria Cristina Nishiwaki-Dantas ${ }^{2}$ \\ Sérgio Felberg"3 \\ Fabiana Amorim ${ }^{4}$ \\ Paulo Elias Correa Dantas ${ }^{5}$
}

\begin{tabular}{|l|}
\hline RESUMO \\
\hline Objetivo: Comparar as alterações quantitativas produzidas no filme \\
lacrimal de pacientes submetidos a ceratectomia fotorrefrativa (PRK) e \\
ceratomileusis in situ (LASIK) ao longo de um ano. Métodos: Desenvol- \\
vemos estudo prospectivo, longitudinal, comparativo, pós-intervencional \\
e não-aleatório para detectar diferenças entre a função lacrimal antes e \\
após a correção de ametropias por excimer laser pelos métodos PRK \\
(Grupo 1= 48 pacientes) e LASIK (Grupo $2=36$ pacientes) usando teste \\
de Schirmer 1 no olho direito e do fio de fenol vermelho no olho esquerdo. \\
Resultados: No período compreendido entre o primeiro e terceiro mês \\
após ambos os procedimentos houve diminuição nos valores das medi- \\
das do teste de Schirmer e do fenol vermelho relativos aos valores basais. \\
Um ano após, os valores voltaram a ser iguais aos valores basais pré- \\
cirúrgicos. Não houve diferença entre os grupos estudados. Conclusão: \\
Apesar da diminuição, dentro dos limites normais, da produção de \\
lágrima reflexa em ambos os grupos, tal alteração parece não trazer \\
problemas maiores em pacientes normais.
\end{tabular}

Descritores: Erros de refração/cirurgia; Ceratectomia fotorrefrativa por excimer laser/ métodos; Córnea/fisiopatologia; Estudos prospectivos; Estudo comparativo
O trabalho foi realizado no Departamento de Oftalmologia da Santa Casa de São Paulo e Hospital Oftalmológico de Sorocaba.

${ }^{1}$ Assistente do Setor de Córnea e Doenças Externas, Departamento de Oftalmologia da Santa Casa de Misericórdia de São Paulo - São Paulo (SP) - Brasil.

${ }^{2}$ Chefe do Departamento de Oftalmologia da Santa Casa de Misericórdia de São Paulo - São Paulo (SP) - Brasil

${ }^{3}$ Coordenador do Ambulatório de Superfície Ocular e Lágrima (SOL), Setor de Córnea e Doenças Externas Departamento de Oftalmologia da Santa Casa de Misericórdia de São Paulo - São Paulo (SP) - Brasil.

${ }^{4}$ Assistente do Setor de Córnea e Doenças Externas, Departamento de Oftalmologia da Santa Casa de Misericórdia de São Paulo - São Paulo (SP) - Brasil.

${ }^{5}$ Chefe do Setor de Córnea e Doenças Externas, Departamento de Oftalmologia da Santa Casa de Misericórdia de São Paulo - São Paulo (SP) - Brasil; Hospital Oftalmológico de Sorocaba - Sorocaba (SP) - Brasil.

Endereço para correspondência: Paulo Elias C. Dantas. Rua Martinico Prado 26 - Conj. 181/182 - São Paulo (SP) CEP 01224-010

Recebido para publicação em 14.01.2006

Última versão recebida em 28.06.2006

Aprovação em 09.07.2006

Os autores não têm interesse comercial sobre produtos/ artigos citados neste texto.

Nota Editorial: Depois de concluída a análise do artigo sob sigilo editorial e com a anuência do Dr. Renato Ambrósio Jr. sobre a divulgação de seu nome como revisor, agradecemos sua participação neste processo.

\section{INTRODUÇÃO}

Com a ablação tecidual induzida pela ação física do excimer laser sobre o estroma anterior da córnea, é possível corrigir erros refrativos pela remodelagem do contorno óptico da superfície anterior da córnea. Ambas as técnicas preconizadas para a correção dessas ametropias, a ceratectomia fotorefrativa (PRK) e a ceratomileusis in situ (LASIK), são consideradas efetivas e seguras e ganham, cada vez mais, maior aceitação no meio oftalmológico como opção terapêutica.

$\mathrm{Na}$ prática diária do atendimento de pacientes submetidos à cirurgia refrativa, é comum depararmo-nos com pacientes que referem sintomas de "sensação de olho seco" após o procedimento cirúrgico refrativo. O mecanismo patogênico que levaria a este queixa clínica não é ainda esclarecido. Uma das possibilidades aventadas seria de que durante a ablação do estroma corneal, ramos de terminações nervosas do plexo subepitelial seriam removidos, induzindo à diminuição transitória da sensibilidade corneal, o que poderia levar a menor produção do conteúdo aquoso da lágrima, por diminuição do estímulo eferente, necessário à sua produção e biodisponibilidade para compor o filme lacrimal ${ }^{(1-6)}$

Em indivíduos normais, a estimulação dos nervos aferentes da superfície ocular induz ao piscar imediato (em média de 12-15 vezes por minuto) e à produção de lágrima reflexa pela glândula lacrimal. O estímulo corneal 
aferente percorre o ramo oftálmico do nervo trigêmio $\left(5^{\circ}\right.$ par craniano), passando pelo gânglio trigeminal e núcleo espinal trigeminal, alcançando a primeira sinapse na ponte. Uma via aferente secundária seria constituída pelos neurônios préganglionares simpáticos da medula espinhal, gânglio cervical superior e cadeia simpática para-vertebral. Após processado na ponte, o estímulo induz resposta eferente através das fibras aferentes da ponte, nervo facial ( $7^{\circ}$ par craniano), gânglio ptérigo-palatino, sinapses e fibras para a glândula lacrimal, ponto final da via aferente primária. As fibras simpáticas do gânglio cervical superior são responsáveis pela resposta pela via aferente secundária junto à glândula lacrimal.

O objetivo deste estudo foi de investigar o efeito do tratamento cirúrgico de ametropias pelos métodos PRK e LASIK sobre a estabilidade do filme lacrimal de pacientes submetidos a estas técnicas por um período de um ano para evidenciar se há diferenças entre as técnicas de correção.

\section{MÉTODOS}

\section{Desenho do estudo}

Desenvolvemos estudo prospectivo, longitudinal, comparativo, pós-intervencional e não-aleatório para detectar diferenças entre a função lacrimal antes e após a correção de ametropias por excimer laser pelos métodos PRK e LASIK.

\section{Seleção de pacientes}

\section{Critérios de inclusão}

Foram incluídos neste estudo, pacientes que procuraram o Setor de Córnea, Doenças Externas e Cirurgia Refrativa do Hospital Oftalmológico de Sorocaba, São Paulo com intenção de se submeter à cirurgia refrativa no período de junho a setembro de 1999. Os pacientes tinham indicação de PRK ou LASIK em função de vários aspectos, tais como, grau da ametropia a ser corrigida, espessura de córnea e opção pessoal pela técnica.

\section{Critérios de exclusão}

- Uso de medicação sistêmica e/ou tópica, com comprovada ação sobre o filme lacrimal;

- Portador de doença sistêmica e/ou ocular, com repercussão sobre a função lacrimal;

- Olho vermelho crônico de qualquer etiologia.

Foram incluídos 48 pacientes candidatos a LASIK (Grupo 1) e 36 pacientes candidatos a PRK (Grupo 2).

Os pacientes do Grupo $1(\mathrm{n}=48)$ apresentavam média de idade de $36,18 \pm 8$ anos, com 22 mulheres $(45,83 \%)$ e 26 homens $(54,16 \%)$, enquanto que o Grupo $2(n=36)$ apresentava média de idade de 35,11 $\pm 9,11$ anos, com 15 mulheres $(41,66 \%)$ e 21 homens $(58,33 \%)$ (Gráficos 1 e 2).

O equivalente esférico dos pacientes do Grupo 1 (LASIK) era de $-4,41 \pm 2,99$ no olho direito e $-4,44 \pm 2,84$ no olho esquerdo, enquanto que no Grupo 2 (PRK) estes valores eram de $-2,35 \pm 0,95$ e $-2,38 \pm 1,17$, respectivamente.

\section{Ética e consentimento livre informado}

O protocolo deste estudo foi submetido ao Comitê de Ética do Hospital Oftalmológico de Sorocaba e aprovado sem restrições. Todos os pacientes incluídos neste estudo, como rotineiramente é feito antes da indicação da cirurgia, eram informados sobre riscos e benefícios da cirurgia, obtinham descrição sucinta (com termos acessíveis à sua condição social e intelectual) da técnica cirúrgica, o tempo de recuperação da visão e dos cuidados gerais após a cirurgia. Ao final dos esclarecimentos, o paciente assinava termo de consentimento livre e informado.

\section{Técnica cirúrgica}

Todas as cirurgias foram feitas pelo mesmo cirurgião (PECD), seguindo técnica padrão de PRK e LASIK, usando o

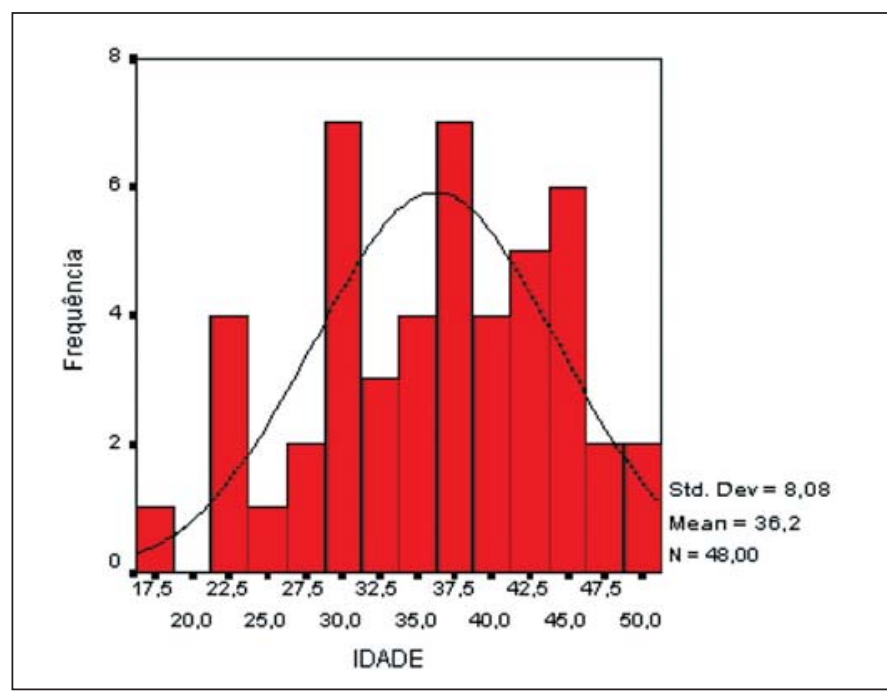

Gráfico 1 - Características demográficas quanto à idade dos pacientes do Grupo 1 (LASIK)

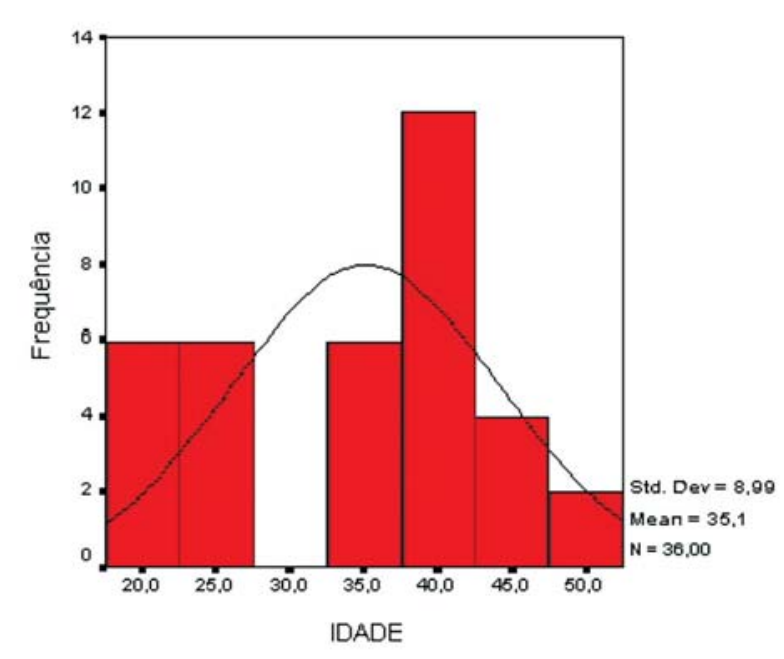

Gráfico 2 - Características demográficas quanto à idade dos pacientes do Grupo 2 (PRK) 
excimer laser Nidek EC 5000 (Nidek, Japão). No PRK, feito com intervalo de sete dias entre o primeiro e o segundo procedimento, utilizamos lente de contato terapêutica gelatinosa por 72 horas até epitelização completa da córnea. A prescrição-padrão após a cirurgia era de colírio de ofloxacino $0,3 \%$ (Oflox ${ }^{\circledR}$, Alcon) de 6 em 6 horas por sete dias, colírio de cetorolaco trometamine (Acular ${ }^{\circledR}$, Alcon) de 6 em 6 horas por dois dias. Após a remoção da lente de contato, era iniciado tratamento com colírio de fluorometolona $0,25 \%$ (Flumex $^{\circledR}$, Alcon) de 6 em 6 horas, com regressão progressiva por um período de oito semanas.

No LASIK, utilizamos o microceratótomo Hansatome (Bausch \& Lomb Surgical, Claremont, EUA) com anel de 8,5 mm de diâmetro e profundidade de "flap" corneal de $180 \mu$ m. A zona de ablação variou de 6 a 7,5 mm, baseada no erro refrativo a corrigir e sua relação com a espessura corneal e diâmetro pupilar. As cirurgias foram feitas em ambos os olhos em um mesmo dia e, após a cirurgia, o paciente era orientado a usar colírio de ofloxacino $0,3 \%$ (Oflox ${ }^{\circledR}$, Alcon) de 6 em 6 horas por sete dias associado ao colírio de fluorometolona $0,25 \%$ (Flumex ${ }^{\circledR}$, Alcon) de 6 em 6 horas por sete dias.

\section{Exames e seguimento dos pacientes}

Os pacientes incluídos neste estudo foram submetidos a exame oftalmológico prévio que incluía a avaliação da acuidade visual com tabela de Snellen, biomicroscopia à lâmpada de fenda, refratometria estática e dinâmica, mapeamento de retina, tonometria pneumática e de aplanação, paquimetria e topografia de córnea. Um dia antes do procedimento cirúrgico, o paciente era submetido a avaliação da função lacrimal com o teste de Schirmer tipo 1 no olho direito, seguido de avaliação do teste da fita de fenol vermelho no olho esquerdo (dez minutos após a avaliação do OD). Os mesmos procedimentos foram repetidos 30 dias, 90 dias e um ano após a cirurgia.

$\mathrm{O}$ teste de Schirmer foi realizado usando-se tiras de papel milimetrado especiais, colocadas no fundo de saco ínfero-lateral por 15 minutos. A distância (em mm) percorrida pela lágrima sobre o papel era medida, anotada e arquivada em um banco de dados (Excel, Microsoft) como o valor do teste de Schirmer 1.

O teste do fio de fenol vermelho (Zone Quick ${ }^{\circledR}$, Menicon Co., Japão) era realizado no olho esquerdo, dez minutos após a realização do teste de Schirmer e consistia na colocação do fio de fenol vermelho no fundo de saco ínfero-lateral por 15 segundos, cronometrados digitalmente (Figura 1). A distância percorrida (em $\mathrm{mm}$ ) pela lágrima sobre o fio era medida, anotada e arquivada como o valor do teste do fenol vermelho (Figura 2).

Durante os testes, mantivemos controle ambiental atmosférico (temperatura ambiente e umidade relativa do ar) com termo-higrômetro digital Oregon Scientific ${ }^{\mathrm{TM}}$, modelo JB913R (Oregon, EUA). Os valores de temperatura (em graus Celsius) e umidade relativa do ar (em percentual) eram anotados antes de cada medida e arquivados na base de dados.

\section{Análise estatística}

\section{Poder estatístico do estudo}

O cálculo do poder estatístico deste estudo foi realizado

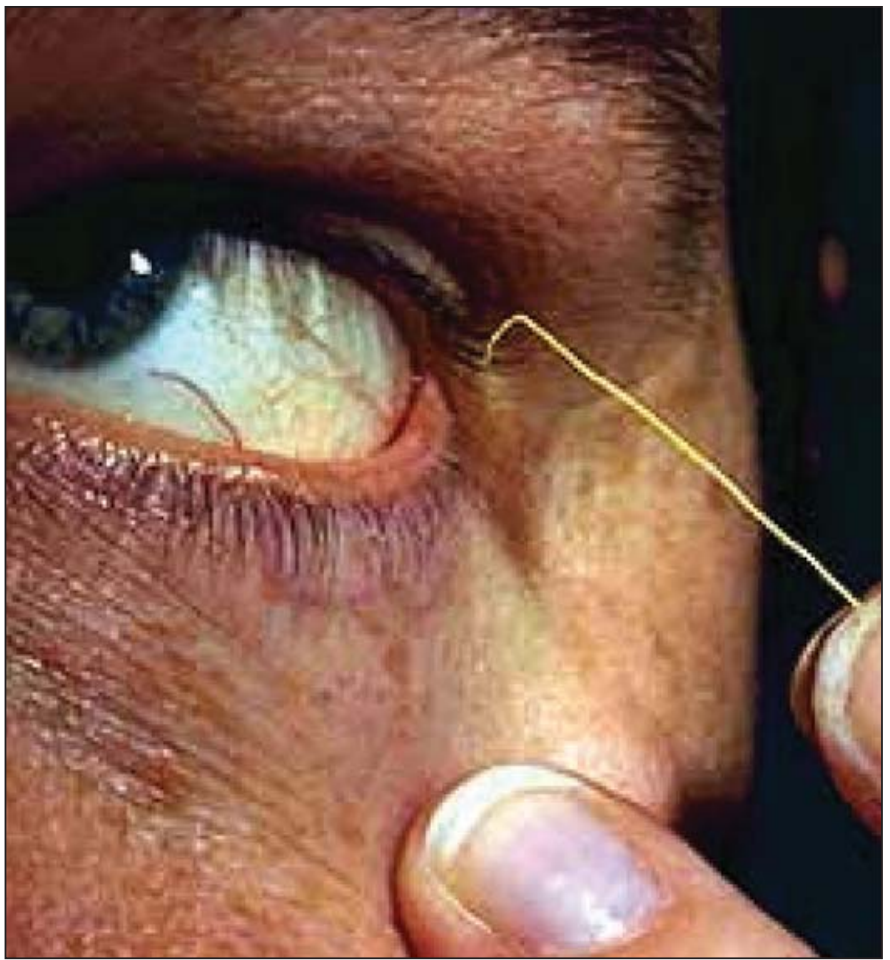

Figura 1 - Colocação do fio de fenol vermelho no 1/3 ínfero-lateral do fundo de saco conjuntival de paciente do Grupo 2

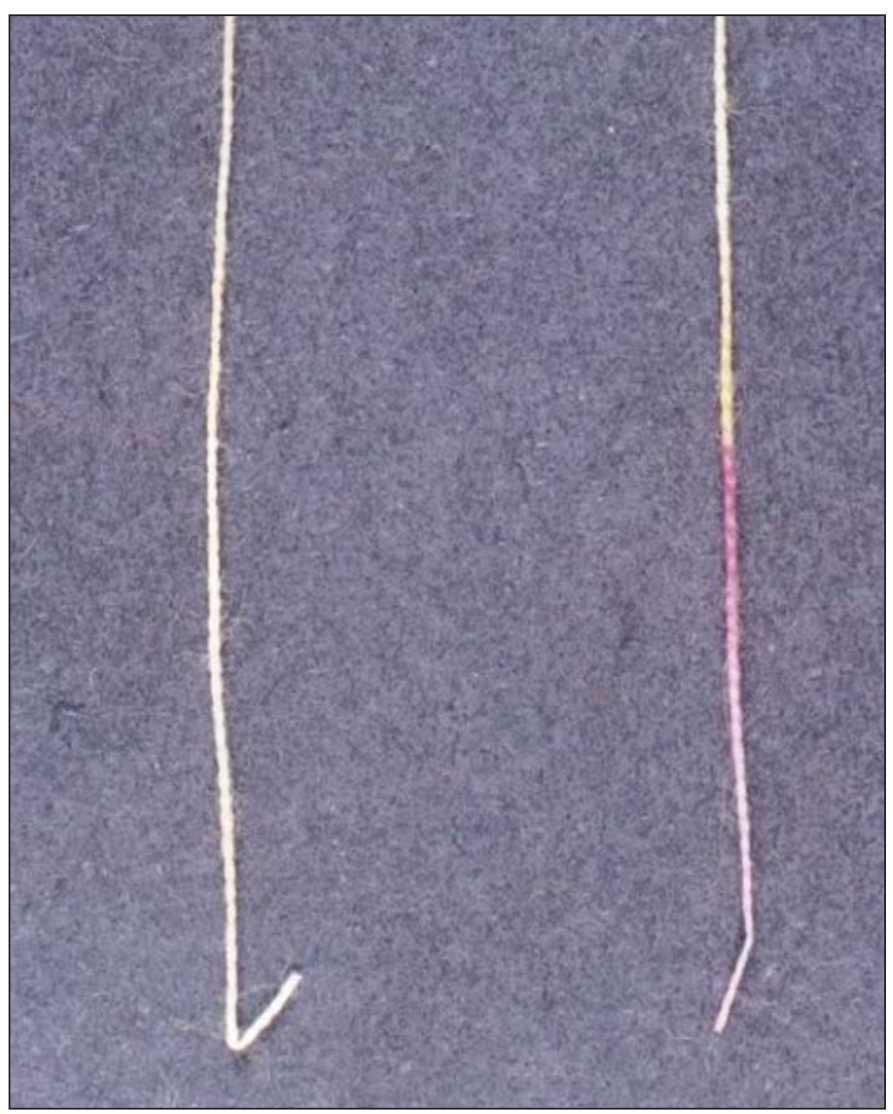

Figura 2 - Tiras de fio de fenol vermelho. Notar a mudança de coloração do fio à direita, após contato com o filme lacrimal por 15 segundos. 
durante a fase de planejamento (protocolo), com o auxílio do programa de computador Power and Sample Size Calculation versão 2.1.23, julho de 2002 (disponível na URL http:// www.mc.vanderbilt.edu/prevmed/os/), assumindo-se que a relevância clínica mínima para o teste de Schirmer 1 e para o teste do fio de fenol vermelho seja de $1 \mathrm{~mm}$, com desviopadrão conservador de $3 \mathrm{~mm}$. Estes dados, quando submetidos aos testes de cálculo de tamanho da amostra e poder estatístico do estudo sugeriram um poder estatístico de $95 \%$, quando 48 pacientes fossem envolvidos.

O teste da análise de variância (ANOVA) foi utilizado para determinar a significância das alterações do teste de Schirmer e do fenol vermelho durante as repetidas medidas.

O teste $\mathbf{t}$ de Student para amostras pareadas foi utilizado para comparar as diferenças das médias de variáveis dicotômicas.

Para todas as análises estatísticas, adotamos nível de significância estatística de $\mathrm{p}<0,05$. Os dados foram processados e analisados com o auxílio do programa (software) Statistical Package for the Social Sciences (SPSS), versão 10.0 (www.spss.com).

\section{RESULTADOS}

A tabela 1 sumariza as variações das medidas (média e desvio-padrão) do teste de Schirmer e fenol vermelho antes, 30 dias, 90 dias e um ano após o procedimento cirúrgico (LASIK) nos pacientes do Grupo 1.

A tabela 2 faz o mesmo com as variações das medidas (média e desvio-padrão) do teste de Schirmer e Fenol vermelho antes, 30 dias, 90 dias e um ano após o procedimento cirúrgico PRK nos pacientes do Grupo 2.

Os gráficos, com a evolução das medidas antes, 30 dias, 90 dias e um ano após as cirurgias podem ser analisados nos anexos (vide anexos 1, 2, 3 e 4).

\section{Grupo 1-LASIK}

Quando submetidos ao teste de análise de variância (ANOVA), os dados dos olhos direitos dos pacientes do Grupo 1 (submetidos a LASIK e teste de Schirmer), mostraram significância estatística $(\mathrm{p}<0,05)$ entre o valor da medida basal e o primeiro e terceiro mês de acompanhamento. Nos olhos esquerdos (submetidos a LASIK e teste do fenol vermelho),

\begin{tabular}{|c|c|c|}
\hline \multirow[b]{2}{*}{ Tempo } & \multicolumn{2}{|c|}{ Teste } \\
\hline & $\begin{array}{l}\text { Schirmer } \\
\text { (no olho direito, } \\
\text { em } \mathrm{mm} \text { ) }\end{array}$ & $\begin{array}{c}\text { Fenol vermelho } \\
\text { (no olho esquerdo, } \\
\text { em } \mathrm{mm} \text { ) }\end{array}$ \\
\hline Antes da cirurgia & $17,27 \pm 9,75$ & $21,20 \pm 5,40$ \\
\hline Um mês após & $14,22 \pm 9,42$ & $19,27 \pm 4,92$ \\
\hline Três meses após & $14,45 \pm 8,92$ & $19,41 \pm 5,40$ \\
\hline Um ano após & $17,18 \pm 9,29$ & $21,20 \pm 5,15$ \\
\hline
\end{tabular}

\begin{tabular}{|c|c|c|}
\hline \multirow[b]{2}{*}{ Tempo } & \multicolumn{2}{|c|}{ Teste } \\
\hline & $\begin{array}{c}\text { Schirmer } \\
\text { (no olho direito, } \\
\text { em } \mathrm{mm} \text { ) }\end{array}$ & $\begin{array}{c}\text { Fenol vermelho } \\
\text { (no olho esquerdo, } \\
\text { em mm) }\end{array}$ \\
\hline Antes da cirurgia & $20,13 \pm 9,46$ & $21,61 \pm 5,44$ \\
\hline Um mês após & $18,91 \pm 8,56$ & $18,77 \pm 6,97$ \\
\hline Três meses após & $17,30 \pm 8,97$ & $16,97 \pm 6,31$ \\
\hline Um ano após & $18,80 \pm 8,41$ & $21,63 \pm 5,08$ \\
\hline
\end{tabular}

os resultados foram semelhantes com significância estatística $(\mathrm{p}<0,05)$ entre o valor da medida basal e o primeiro e terceiro mês de acompanhamento.

Para caracterizarmos o intervalo de tempo com diferença estatisticamente significante (em relação às medidas prévias à cirurgia), compararmos, com teste $\mathbf{t}$ de Student para amostras pareadas, os intervalos de tempo de acompanhamento (um mês, três meses e um ano) com os valores basais obtidos antes da cirurgia. Verificamos nível de significância estatística de $\mathrm{p}<0,05$ no primeiro mês e terceiro mês após a cirurgia. Não houve diferença estatística significante após um ano ( $p>0,05)$.

\section{Grupo 2-PRK}

Quando submetidos ao teste de análise de variância (ANOVA), os dados dos olhos direitos dos pacientes do Grupo 2 (submetidos a PRK e teste de Schirmer), mostraram significância estatística $(p<0,05)$ entre o valor da medida basal e o primeiro e terceiro mês de acompanhamento, acontecendo o mesmo com os olhos esquerdos (submetidos a PRK e teste do fenol vermelho).

Ao compararmos estatisticamente, com teste $\mathbf{t}$ de Student para amostras pareadas, os intervalos do acompanhamento (um mês, três meses e um ano) com os valores basais obtidos antes da cirurgia, verificamos nível de significância estatística de $\mathrm{p}<0,05$ no terceiro mês nos olhos direitos (PRK e teste de Schirmer), não havendo diferença estatística no primeiro mês e um ano após. Nos olhos esquerdos (PRK e teste do fenol vermelho), houve significância estatística $(\mathrm{p}<0,05)$ no primeiro e terceiro mês após o procedimento, não havendo diferença estatística um ano após a cirurgia.

\section{Grupo 1 vs Grupo 2}

Quando comparadas aos pares com teste $\mathbf{t}$ de Student para amostras pareadas, as medidas de ambos os procedimentos mostraram-se homogêneas em todas as fases ( $p>0,05)$, com exceção do teste de Schirmer a um mês em ambos os grupos, que mostrou significância estatística $(\mathrm{p}<0,05)$.

\section{Controle ambiental}

Durante o procedimento a temperatura local foi de 24,59 $\pm 1,15$ ( 22 a $27^{\circ}$ Celsius) e umidade relativa do ar de $59,15 \pm 6,13$ (50 a $78 \%)$. 


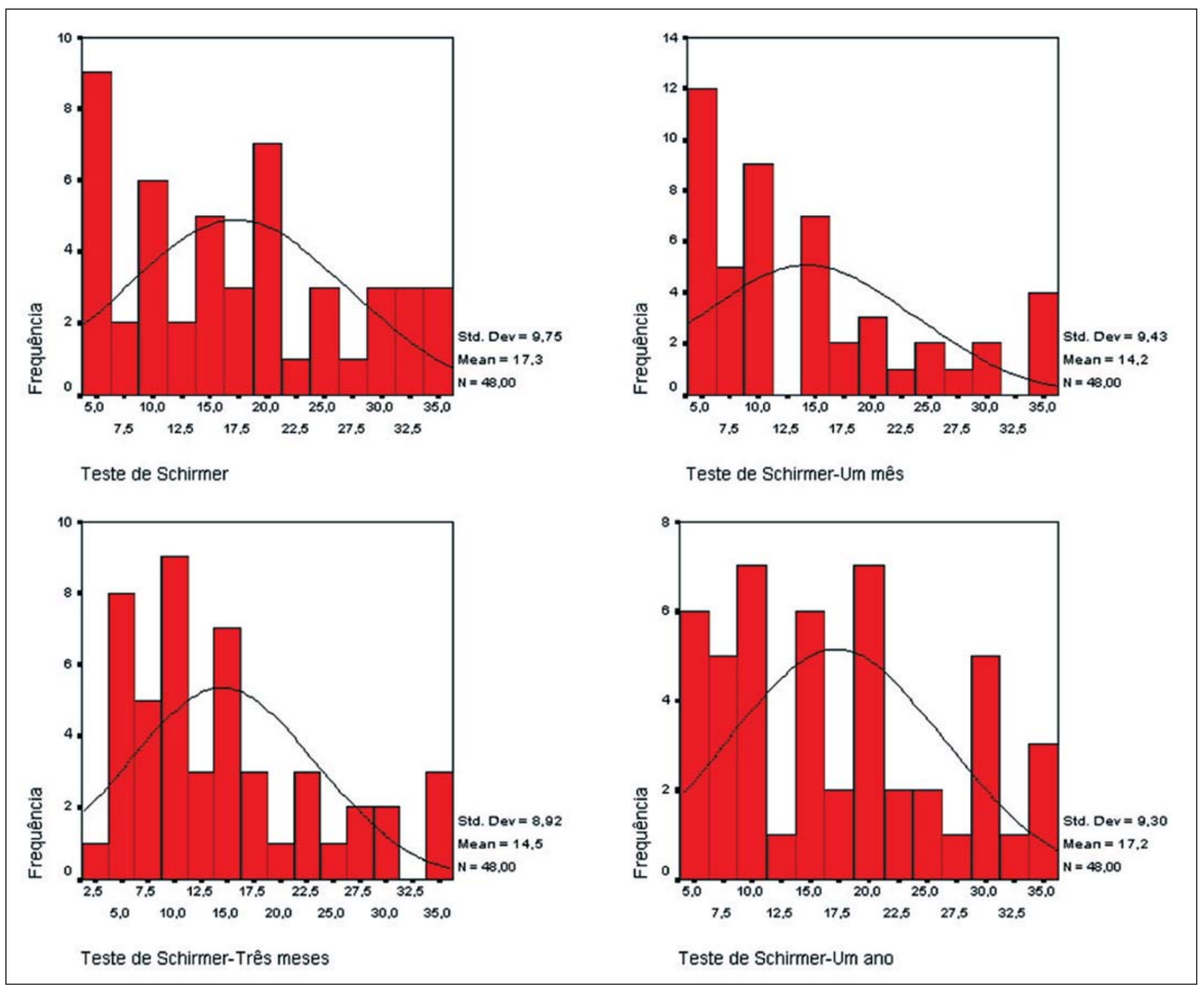

Anexo 1 - Histogramas com valores de média e desvio-padrão dos valores obtidos nos testes de Schirmer em pacientes submetidos à cirurgia refrativa (LASIK) do Grupo 1, antes, um mês, três meses e um ano após

\section{COMENTÁRIOS}

Os resultados obtidos neste estudo parecem confirmar a impressão clínica de que queixas de "sensação olho seco" são comuns após cirurgia refrativa, seja LASIK ou PRK, nas fases iniciais da recuperação cirúrgica. Apesar desses achados apresentarem relevância clínica, principalmente se levarmos em consideração que parte da população que busca correção para erros refrativos pode apresentar olho seco de qualquer etiologia, esta variação quantitativa parece não provocar instabilidade no filme lacrimal como demonstrado por Aras et $\mathrm{al}^{(1)}$, que também identificaram alterações nos valores do teste de Schirmer em pacientes alto míopes submetidos a LASIK, porém sem alterações significantes no tempo de ruptura do filme lacrimal (BUT).
Uma das hipóteses mais prováveis para a variação quantitativa na produção do filme lacrimal estaria relacionada à diminuição da sensibilidade da superfície anterior da córnea, uma vez que, em ambas as técnicas, nervos subepiteliais são lesados durante a fotoablação ${ }^{(1-6)}$. Nos olhos tratados com PRK, a reparação dos nervos epiteliais se inicia da margem da área de ablação em direção ao centro da córnea, completando-se em torno de 6 a 8 meses $^{(6)}$. Já nos olhos tratados com LASIK, a regeneração dos plexos subepiteliais parece estar completa 6 meses após o procedimento ${ }^{(6)}$, seguindo o mesmo direcionamento de reparação do PRK. Matsui et al. ${ }^{(4)}$ pesquisando a sensibilidade da córnea de pacientes submetidos a cirurgia refrativa (PRK e LASIK) com estesiômetro de Cochet-Bonett, perceberam diminuição da sensibilidade da superfície anterior da córnea por pelo menos três meses, tempo de seguimento 


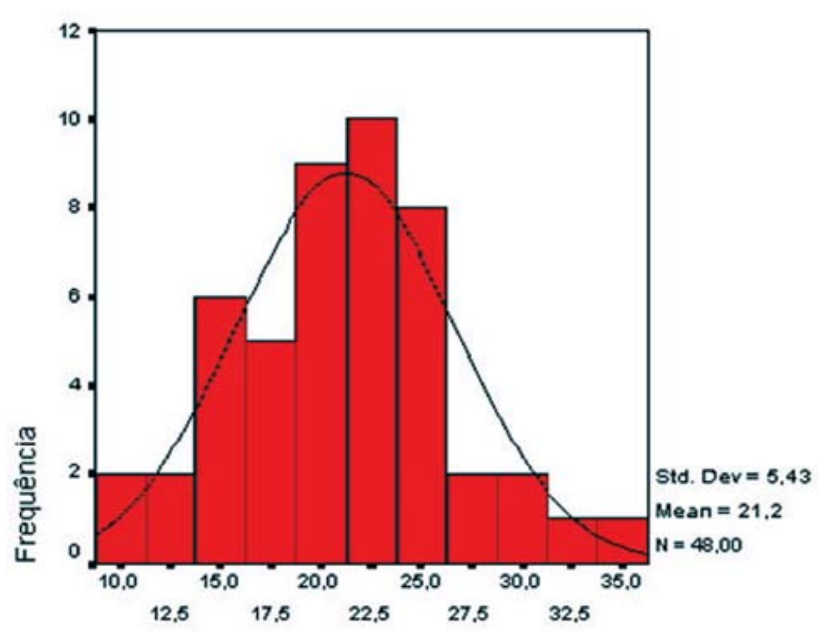

Teste do fenolvermelho

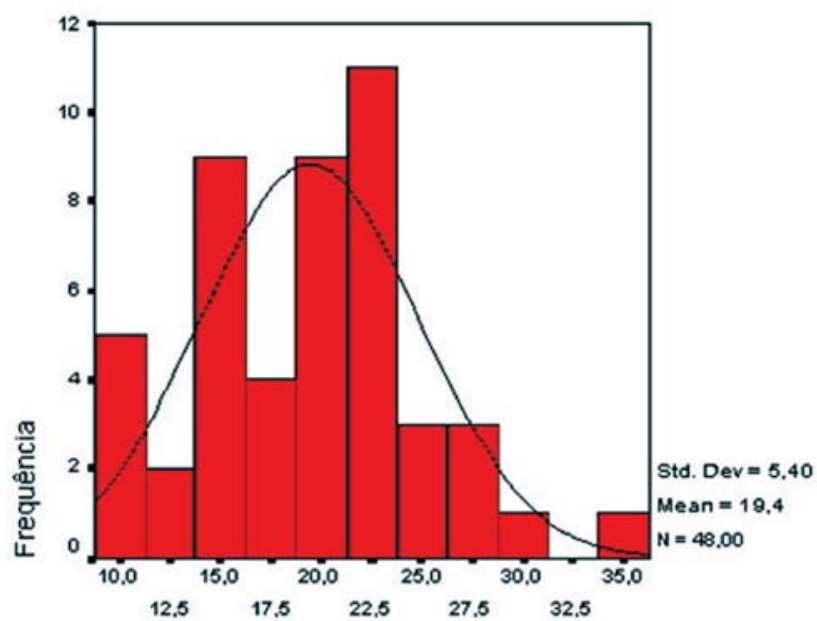

Teste do fenolvermelho-Três meses

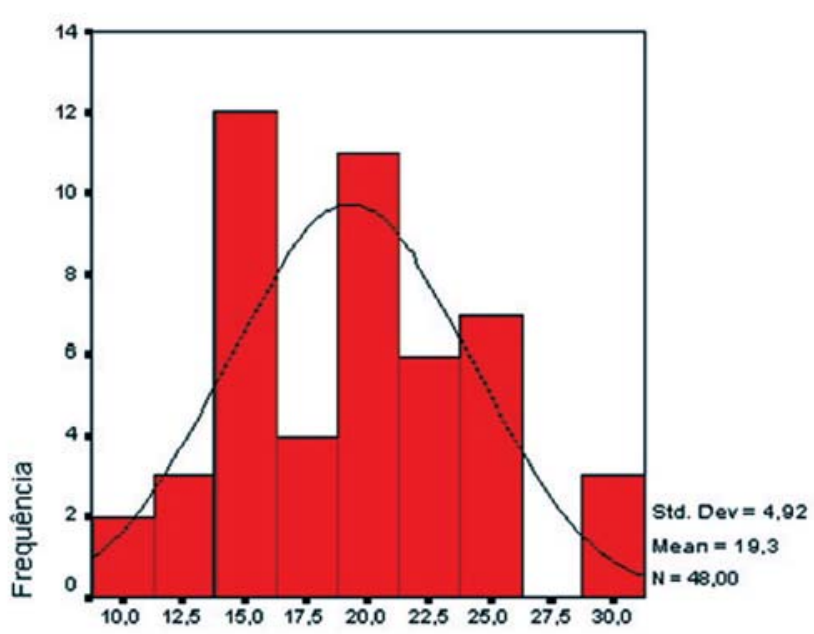

Teste do fenolvermelho-Ummês

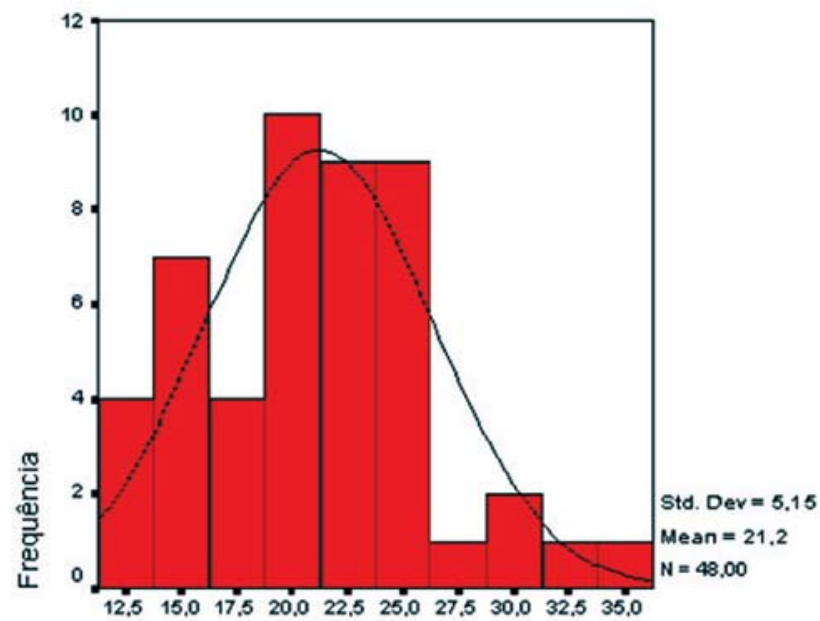

Teste do fenolvermelho-Umano

Anexo 2 - Histogramas com valores de média e desvio-padrão dos valores obtidos nos testes de fenol vermelho em pacientes submetidos à cirurgia refrativa (LASIK) do Grupo 1, antes, um mês, três meses e um ano após

dos pacientes envolvidos no estudo. Estes achados, anteriores aos nossos resultados, poderiam explicar a variação cíclica encontrada em nossas observações, onde, um mês e três meses após o procedimento, identificamos redução significativa da produção de lágrima, com retorno aos valores basais do período pré-operatório, um ano após o procedimento.

O impacto destes achados sobre a população normal parece ser mínimo, e é expresso por queixas subjetivas e sazonais de sensação de corpo estranho, sem maior prejuízo aparente à saúde ocular do paciente, visto ser temporário. Porém, serve de alerta para que nossa avaliação e triagem previa à cirurgia refrativa não se restrinja tão somente aos aspectos de refração, espessura e curvatura da superfície anterior da córnea. Devemos incluir também a busca, através da história clínica do paciente e de minucioso exame biomicroscópico com corantes vitais, de possíveis alterações prévias do filme lacrimal. Certa- mente, pacientes com doenças do colágeno (principalmente a artrite reumatóide do adulto), doenças cicatriciais da superfície ocular (como a dérmato-cérato-conjuntivite atópica) e história de olho vermelho crônico devem ser colocados sob suspeita e ter sua superfície ocular (filme lacrimal incluso) avaliada por meio dos testes específicos de detecção da homeostase do filme lacrimal, como teste de Schirmer, teste do fenol vermelho, tempo de ruptura do filme lacrimal (invasivo ou não), teste da rosa bengala, teste do "clearance" da fluoresceína, para citar os menos dispendiosos financeiramente e fáceis de realizar no consultório de qualquer oftalmologista, sem necessidade de maior aparato tecnológico. O teste do fenol vermelho já demonstrou ser útil em avaliações epidemiológicos de quantificação do filme lacrimal na população brasileira ${ }^{(7)}$.

Uma vez detectado distúrbio do filme lacrimal e avaliada sua magnitude e significado clínico, o cirurgião deverá, nos 


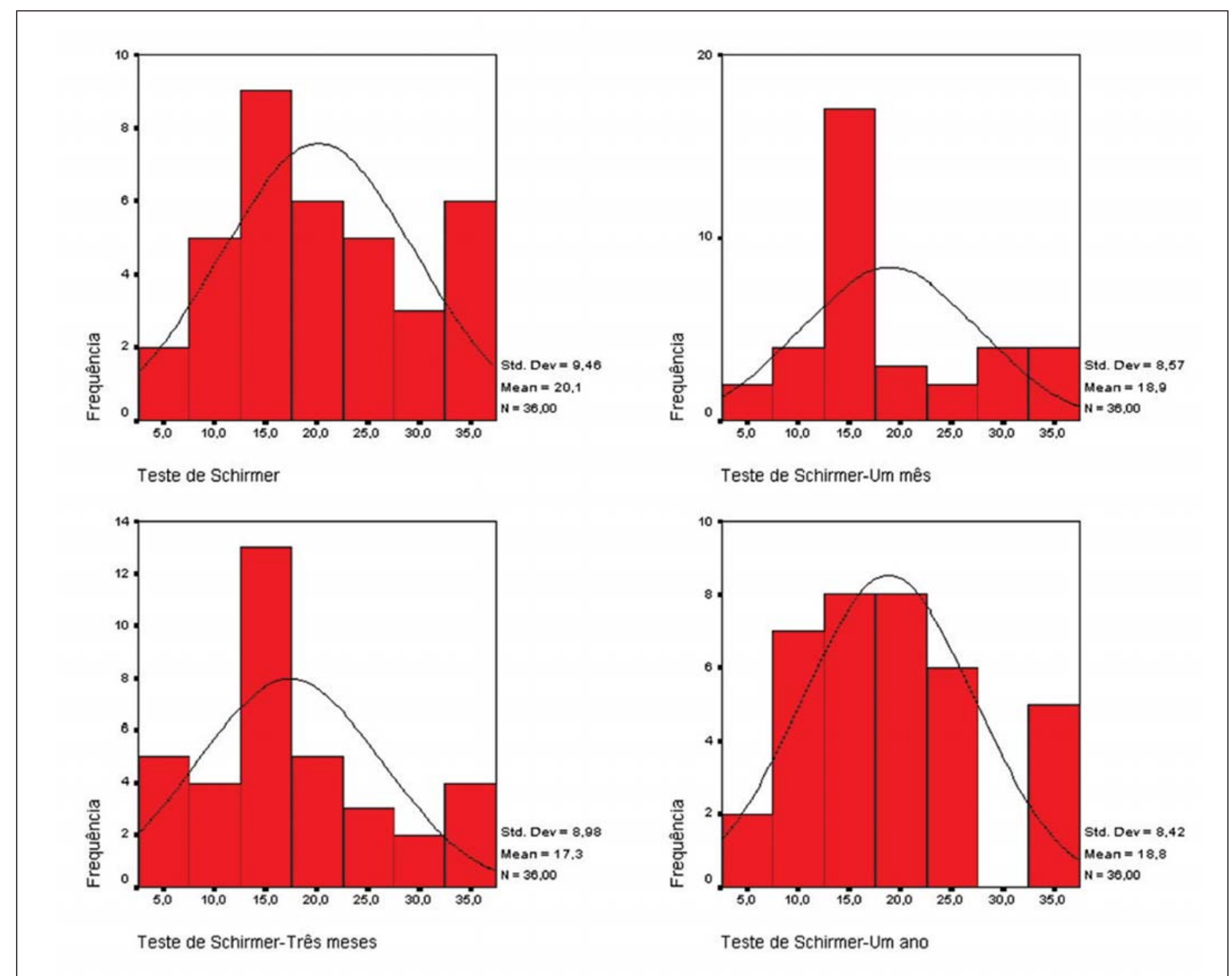

Anexo 3 - Histogramas com valores de média e desvio-padrão dos valores obtidos nos testes de Schirmer em pacientes submetidos à cirurgia refrativa (PRK) do Grupo 2, antes, um mês, três meses e um ano após

casos de olho seco severo, contra-indicar o procedimento e explicar de maneira didática ao paciente as razões de tal conduta, além de orientá-lo quanto ao tratamento específico do problema. Wilson ${ }^{(8)}$ descreveu epiteliopatia neurotrófica presumida em pacientes submetidos à cirurgia refrativa (LASIK) e alerta que essa desordem tende a ser mais comum e severa em pacientes com olho seco pré-existente.

Apesar da diminuição, dentro dos limites normais, da produção de lágrima reflexa, tal alteração parece não trazer problemas maiores em pacientes normais. Suplementação com lágrimas artificiais sem conservantes em pacientes sintomáticos pode ser necessária.

Os dados obtidos com este estudo, evidenciaram diminuição na quantificação do filme lacrimal no primeiro e terceiro mês após realizada a cirurgia refrativa (LASIK e PRK); porém, não se evidenciou distúrbio ou doença da superfície ocular, que fosse caracterizada como iatrogênica. Tal alteração não foi evidenciada um ano após o procedimento, fazendo-nos crer que houve estabilização do filme lacrimal, com valores equiparados aos pré-cirúrgicos.

\section{ABSTRACT}

Purpose: To compare quantitative changes induced in the tear film of patients submited to photorefractive keratectomy and LASIK over a year. Methods: A prospective, longitudinal, comparative, post-interventional study was designed to distinguish tear film changes before and one year after refractive surgery procedures in 48 patients submited to PRK and 36 patients submited to LASIK, using type 1 Schirmer test in the right eye and red phenol thread test in the left eye. Results: 


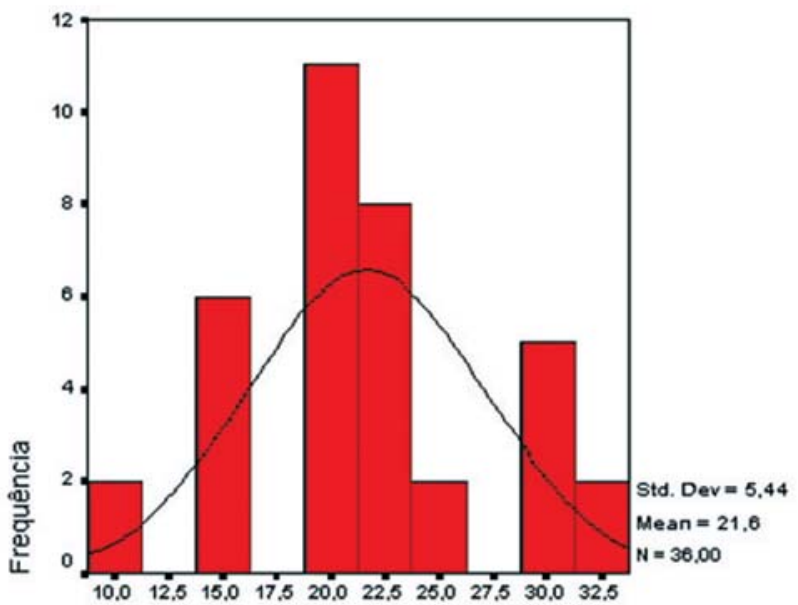

Teste do fenolvermelho

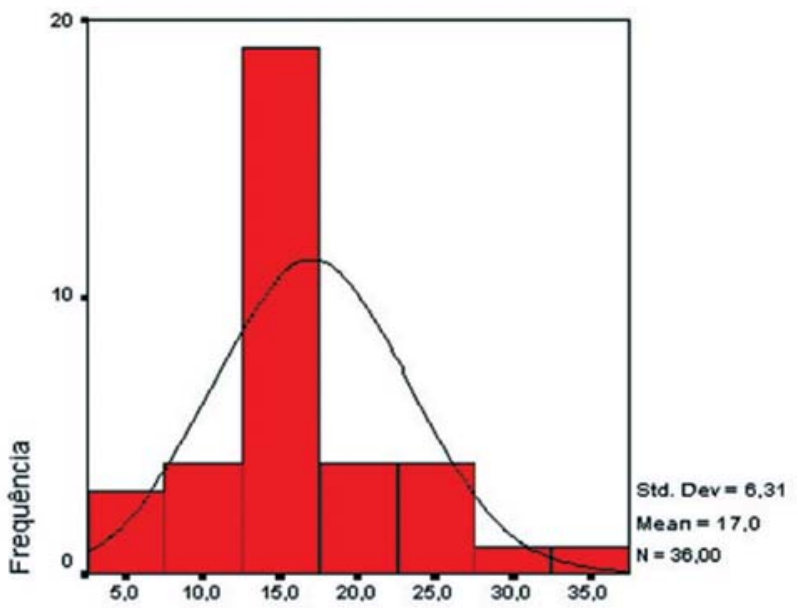

Teste do fenol vermelho - Três meses

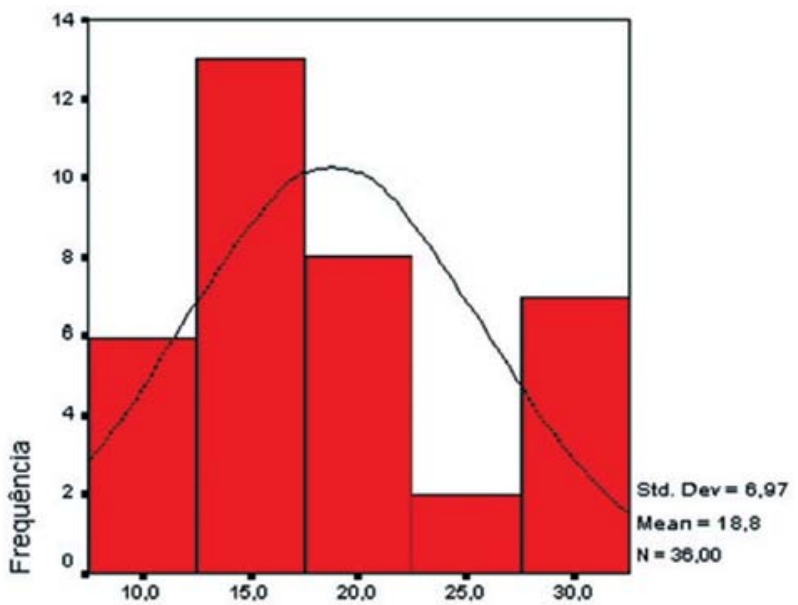

Teste do fenol vermelho-Um mês

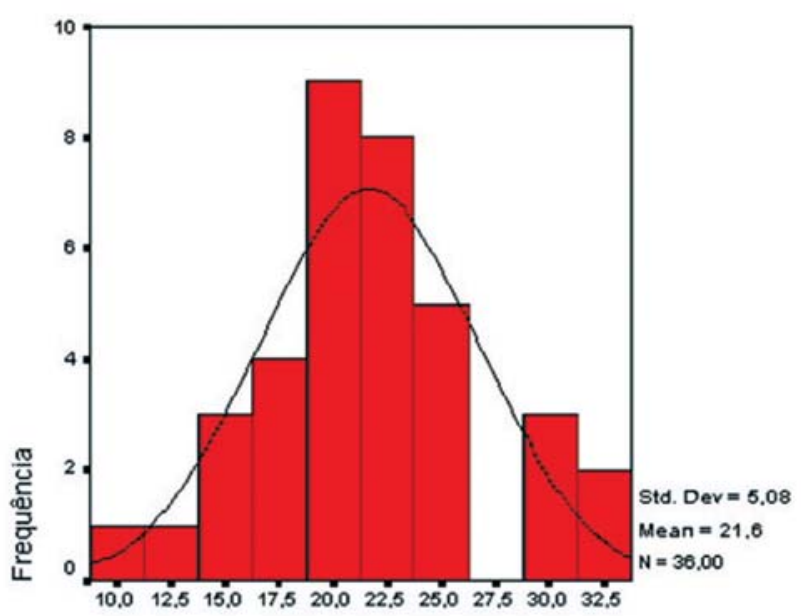

Teste do fenol vermelho-Umano

Anexo 4 - Histogramas com valores de média e desvio-padrão dos valores obtidos nos testes de fenol vermelho em pacientes submetidos à cirurgia refrativa (PRK) do Grupo 2, antes, um mês, três meses e um ano após

Tear film assessment between the first and third postoperative month demonstrated Schirmer and phenol red measurements below basal values for both procedures. One year after the procedures, measurements returned to presurgical values. Conclusion: Besides momentary and within normal limit changes in the measurement of tear film, no complications were found in normal patients.

Keywords: Refractive errors/surgery; Keratectomy, photorefractive, excimer laser/methods; Cornea/physiopathology; Prospective studies; Comparative study

\section{REFERÊNCIAS}

1. Aras C, Ozdamar A, Bahcecioglu H, Karacorlu M, Sener B, Ozkan S. Decrea- sed tear secretion after laser in situ keratomileusis for high myopia. J Refract Surg. 2000;16(3):362-4.

2. Battat L, Macri A, Dursun D, Pflugfelder SC. Effects of laser in situ keratomileusis on tear production, clearance, and the ocular surface. Ophthalmology. 2001;108(7):1230-5

3. Linna TU, Peres-Santonja JJ, Tervo KM, Sakla HF, Alio y Sanz JL, Tervo TM. Recovery of corneal nerve morphology following laser in situ keratomileusis. Exp Eye Res. 1998;66(6):755-63.

4. Matsui H, Kumano Y, Zushi I, Yamada T, Matsui T, Nishida T. Corneal sensation after correction of myopia by photorefractive keratectomy and laser in situ keratomileusis. J Cataract Refract Surg. 2001;27(3):370-3.

5. Ozdamar A, Aras C, Karakas N, Sener B, Karacorlu M. Changes in tear flow and tear film stability after photorefractive keratectomy. Cornea. 1999;18(4):437-9.

6. Kauffmann T, Bodanowitz S, Hesse L, Kroll P. Corneal reinnervation after photorefractive keratectomy and laser in situ keratomileusis: an in vivo study with a confocal videomicroscope. Ger J Ophthalmol. 1996;5(6):508-12.

7. Hida RY, Nishiwaki-Dantas MC, Hida MM, Tsubota K. Estudo quantitativo da lágrima pelo teste do fenol vermelho na população brasileira. Arq Bras Oftalmol. 2005;68(4):433-7.

8. Wilson SE. Laser in situ keratomileusis-induced (presumed) neurotrophic epitheliopathy. Ophthalmology. 2001;108(6):1082-7. 